\title{
Transport properties of coherent matter waves : superfluidity \& Anderson localization
}

\author{
Patricio Leboeuf \\ Laboratoire de Physique Théorique et Modèles Statistiques \\ Université Paris $11 \&$ CNRS, Orsay \\ In collaboration with: \\ M. Albert, S. Moulieras \\ T. Paul, N. Pavloff, K. Richter, P. Schlagheck
}




\title{
Transport properties of coherent matter waves : superfluidity \& Anderson localization
}

\author{
Patricio Leboeuf
}

Laboratoire de Physique Théorique et Modèles Statistiques

Université Paris 11 \& CNRS, Orsay
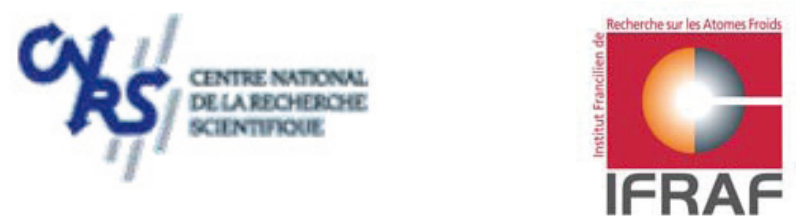

In collaboration with:

M. Albert, S. Moulieras

T. Paul, N. Pavloff, K. Richter, P. Schlagheck 


\section{Light/matter interaction: mechanical effects}

Fundamental process: momentum transfer between photons and atoms

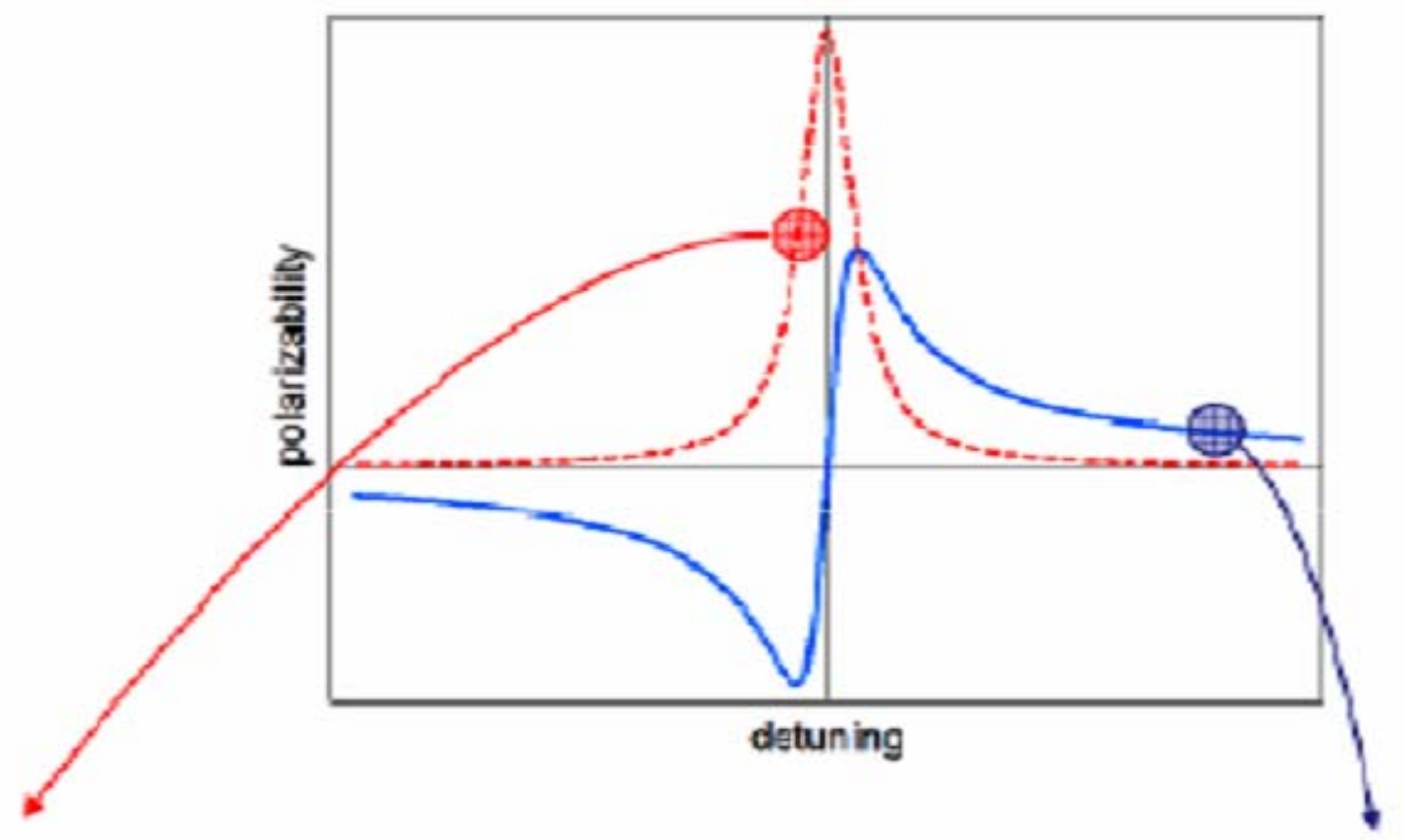

Quasi-resonant interaction:

- absorption (+ spontaneous emission)

- dissipative force (e.g. $\vec{F}=-\gamma \vec{v}$ )

Laser cooling

(MOT, optical molasses, ...)
Non-resonant interaction:

- dispersive interaction

- conservative force $\vec{F}=-\nabla U_{\text {dip }}$ trapping (optical traps, optical lattices, ...) 


\section{Optical trapping}

Far off resonance light induces an electric dipole

$$
\overrightarrow{\mathrm{p}}=\alpha \overrightarrow{\mathrm{E}}
$$

The atomic induced electric dipole then interacts with the e.m. wave

$$
\mathrm{U}(\overrightarrow{\mathrm{r}})=-\vec{p} \cdot \overrightarrow{\mathrm{E}}(\overrightarrow{\mathrm{r}})
$$

Optical trapping 


\section{Transport of a Bose Einstein Condensate}

$\underline{\text { Multipole Oscillations }}$

(sudden offset of the trap)
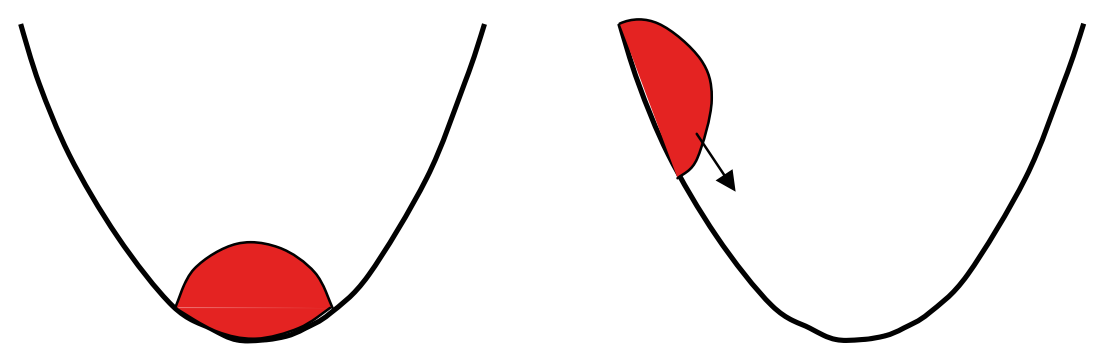

«

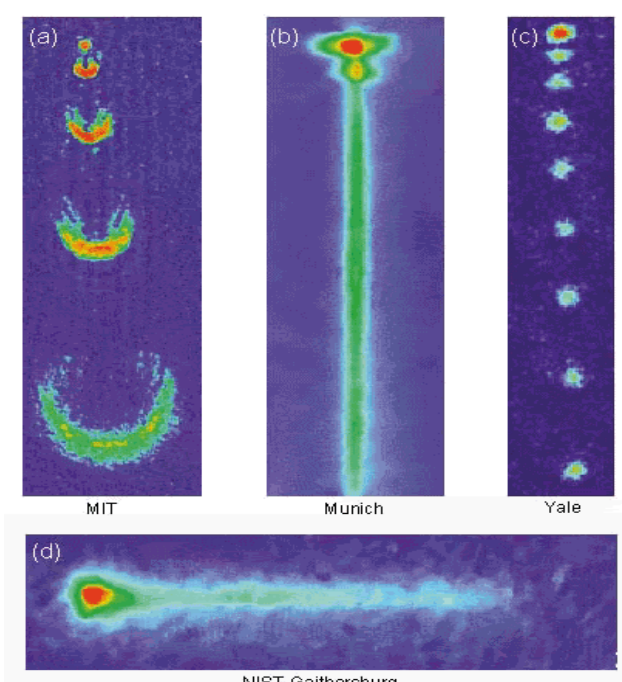

NIST-Gaithersburg

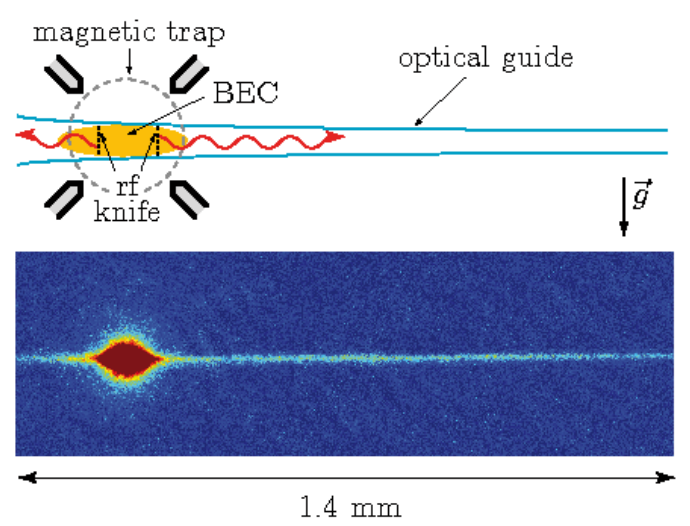

$\underline{\text { Atoms chip }}$

Guerin, Riou, Gaebler, Josse, Bouyer, Aspect, PRL 97 (2006)
《 Stir 》

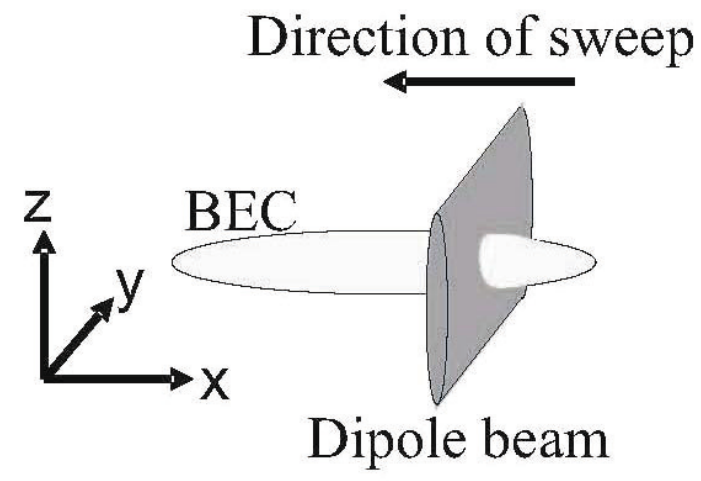

Dipole beam

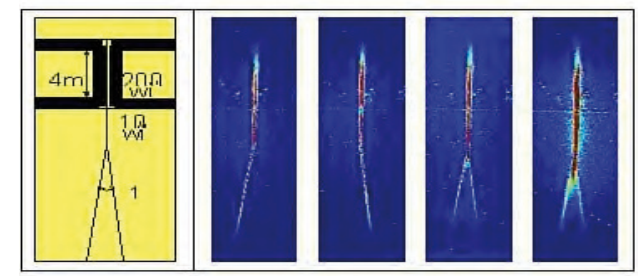


Equations of motion, 1D regime:

$a_{s c} m \omega_{\perp} / \hbar<n a_{s c}<1 \quad a_{s c}: \mathrm{s}$ - wave scattering length $(>0)$

Order parameter: $\quad \psi(x, t) e^{-i \mu t / \hbar}$

$$
i \hbar \partial_{t} \psi=-\frac{\hbar^{2}}{2 m} \partial_{x}^{2} \psi+\left[U(x-V t)+g|\psi|^{2}-\mu\right] \psi
$$

Direction of sweep

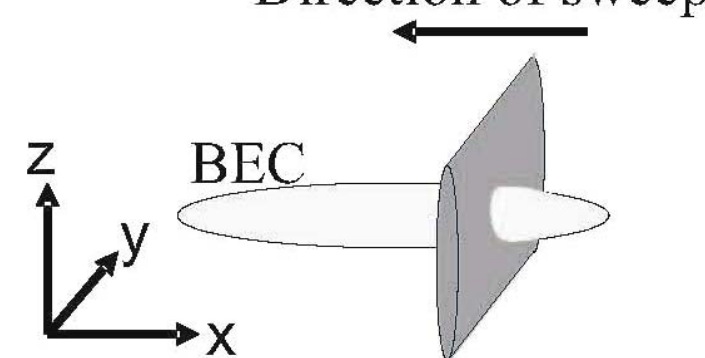

Dipole beam

$\Psi(\vec{r}, t)=\psi(x, t) \phi\left(\vec{r}_{\perp}, n\right) e^{-i \mu t / \hbar}$

$V_{\perp}\left(\vec{r}_{\perp}\right)=\frac{1}{2} \omega_{\perp}^{2} r_{\perp}^{2}$
○ $n(x, t)=\int d^{2} r_{\perp}|\Psi|^{2}=|\psi(x, t)|^{2}$

- $g=2 \hbar \omega_{\perp} a_{s c}$

- $U(x), \quad U(|x| \rightarrow \infty)=0$

$\bigcirc \mu$
Longitudinal density

Interaction parameter

Longitudinal potential

Chemical potential 
$\underline{\text { Stationary transmission modes }}$

$$
\Longrightarrow \psi(x, t)=\psi(X=x-V t)
$$

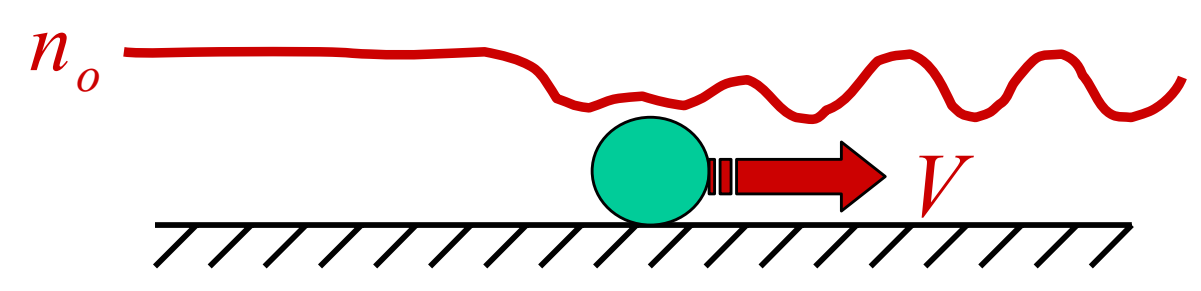

$$
\Longrightarrow\left\{\begin{array}{l}
\mu=g n_{o} \\
c=\sqrt{\mu / m} \\
\xi=\hbar /(m c)
\end{array}\right.
$$

Chemical potential

Speed of sound

Healing length

$$
\psi(X)=\sqrt{n(X)} e^{i S(X)}\left\{\begin{array}{l}
A=\sqrt{n / n_{o}} \\
v=(\hbar / m) \partial_{X} S
\end{array}\right.
$$

$$
\left\{\begin{array}{l}
(v-V) n=J_{\infty} \\
U \frac{d A^{2}}{d X}=\frac{d}{d X}\left[\frac{\hbar^{2}}{2 m}\left(\frac{d A}{d X}\right)^{2}+W(A)\right]
\end{array}\right.
$$

\section{Boundary conditions}$$
\psi(X \rightarrow-\infty)=\sqrt{n_{o}}
$$ 
$\underline{\text { Free modes }}$

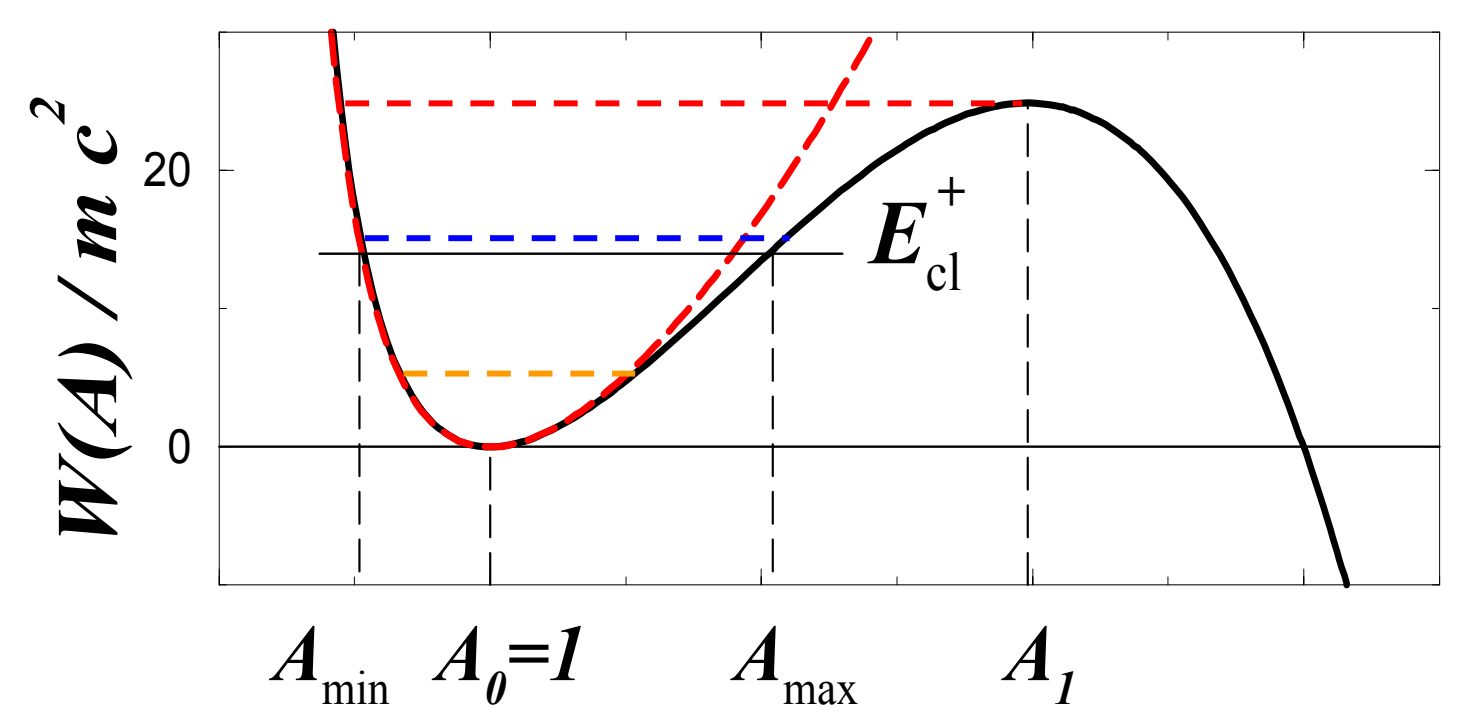

$\underline{\text { Transmission coefficient }}$

$$
\begin{gathered}
A^{2}(X)=1+\delta \rho(X) \quad|\delta \rho|<<\left|\frac{V^{2}}{c^{2}}-1\right| \\
T=\frac{1}{1+m E_{c l}^{+} /\left(2 \hbar^{2} \kappa^{2}\right)}
\end{gathered}
$$
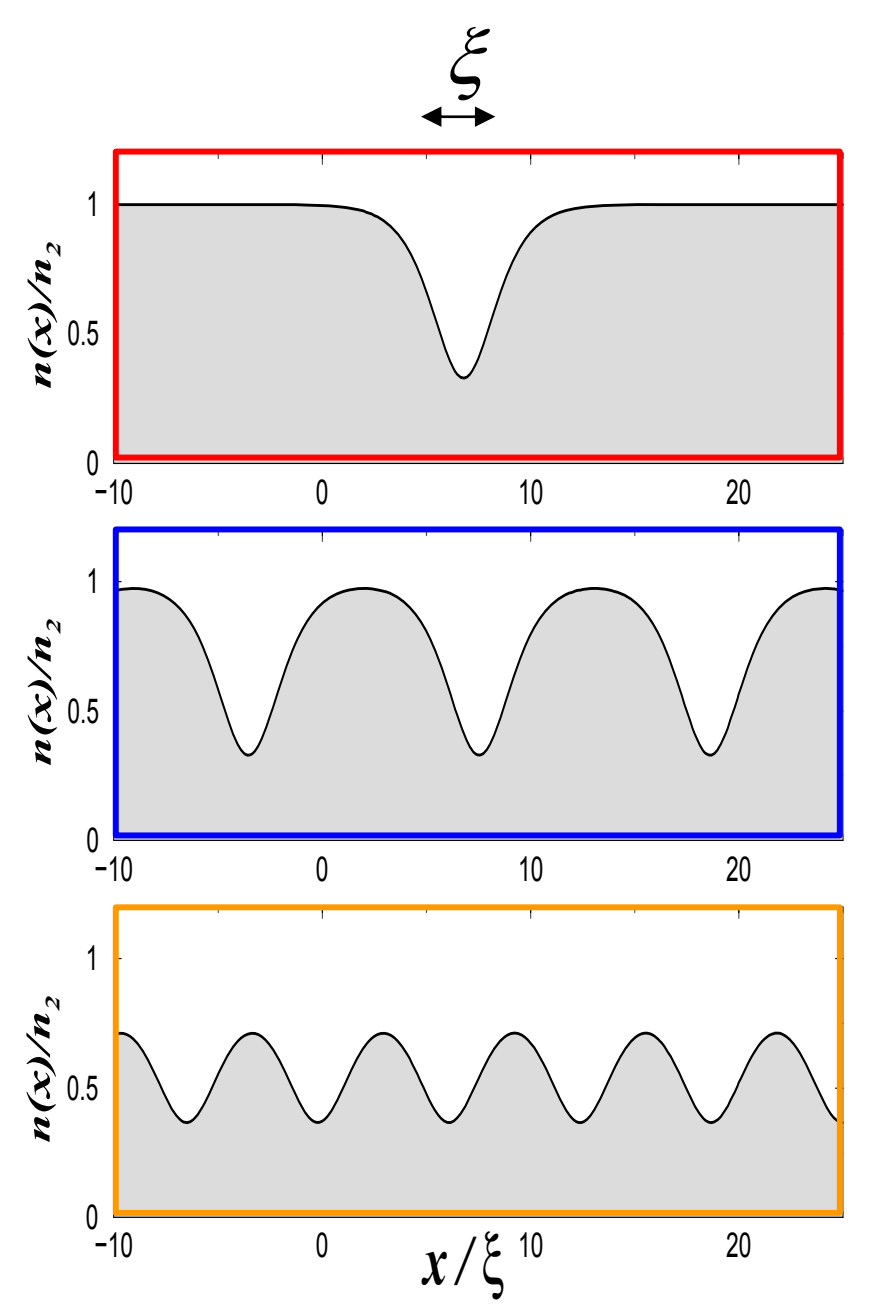

$$
\kappa=\frac{m}{\hbar} \sqrt{\left|V^{2}-c^{2}\right|}
$$




\section{Superfluidity}

- Superfluidity is a phase of matter in which unusual physical effects are observed

- No viscosity (frictionless flow)

- quantized vortices that persist forever

- zero thermodynamic entropy

- infinite thermal conductivity (no temperature gradient can exist)

- fountain effect

- superfluidity and superconductivity are low temperature quantum mechanical effects

- The potential applications of superfluids seem more limited compared to those of superconductors 


\section{Landau criterion}

Assume we have a SF moving at velocity $v$. In order to create an excitation of the fluid (« heat »), the fluid must have enough energy. Suppose we create an excitation of momentum $p$ and energy $\varepsilon(p)$ (dispersion relation). The fluid looses momentum (and energy), with

$$
p=M \delta v
$$

where $\delta v$ the velocity variation and $M$ the fluid mass. The energy variation of the fluid is

$$
\delta E=(1 / 2) M 2 v \delta v=M v \delta v=v p
$$

The excitations are then possible if $\delta E \geqslant \varepsilon(p)=>v p \geqslant \varepsilon(p)$

$$
v \geq v_{c}^{L}=\min \left(\frac{\varepsilon(p)}{p}\right)
$$

$$
\begin{gathered}
\Rightarrow \text { if } \quad \varepsilon(p)=\frac{p^{2}}{2 m} \rightarrow \quad v_{c}^{L}=0 \\
\text { Interactions! }
\end{gathered}
$$

\section{Landau's (energetical) instability of the superflow}

\section{Linear analysis of the stability of the uniform flow under a small perturbation}

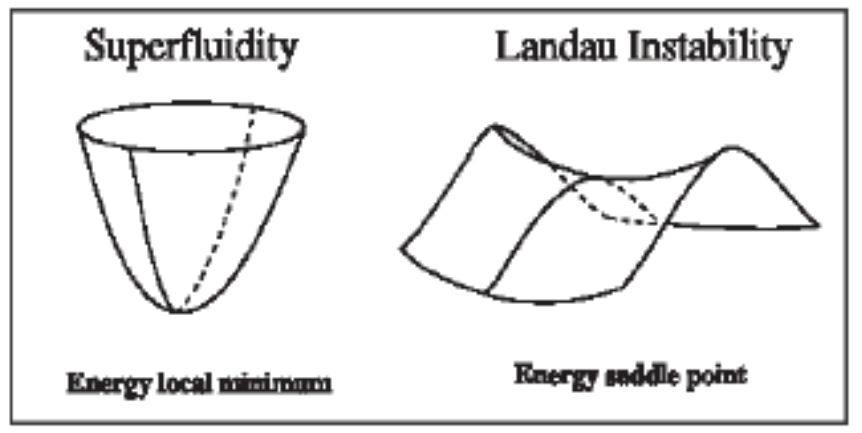

$$
\begin{gathered}
\psi(x)=\phi_{0}(x)+\delta \phi(x) \\
\Rightarrow \delta E=\int d x\left(\delta \phi, \delta \phi^{*}\right) \mathcal{M}\left(\begin{array}{c}
\delta \phi \\
\delta \phi^{*}
\end{array}\right) \\
\text { - Eigenvalues of } \mathcal{M}<0 \text { ? }
\end{gathered}
$$




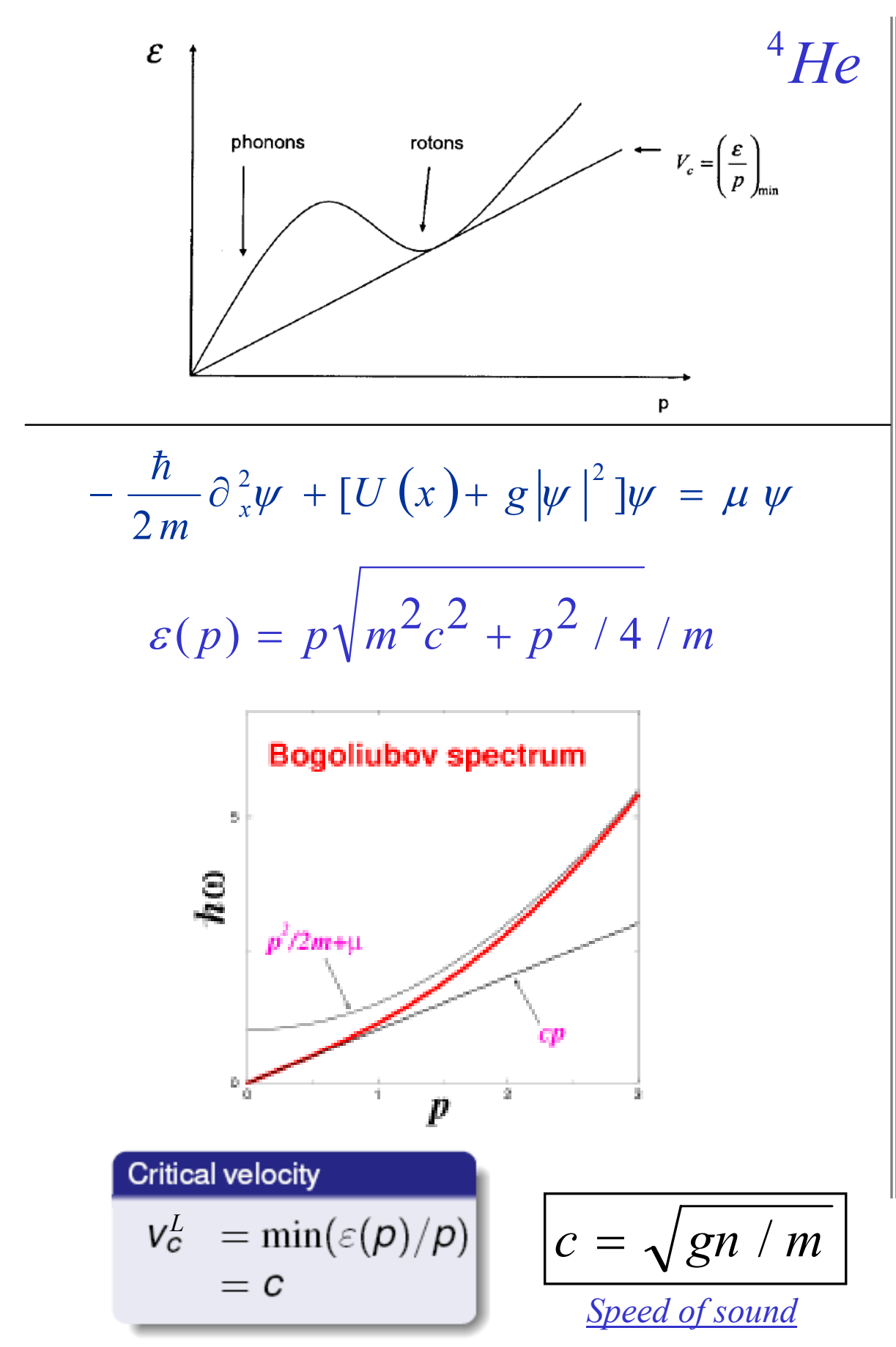

\section{$\underline{\text { Superfluidity }}$}

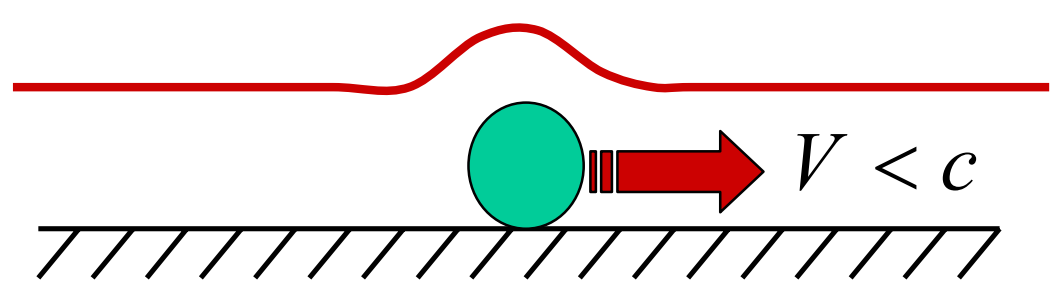

- No dissipation

- No drag force

- Perfect transmission

- Normal fluid fraction

In measurements, $\quad v_{c} \leq v_{c}^{L}$ 


\section{Flow past an impurity}

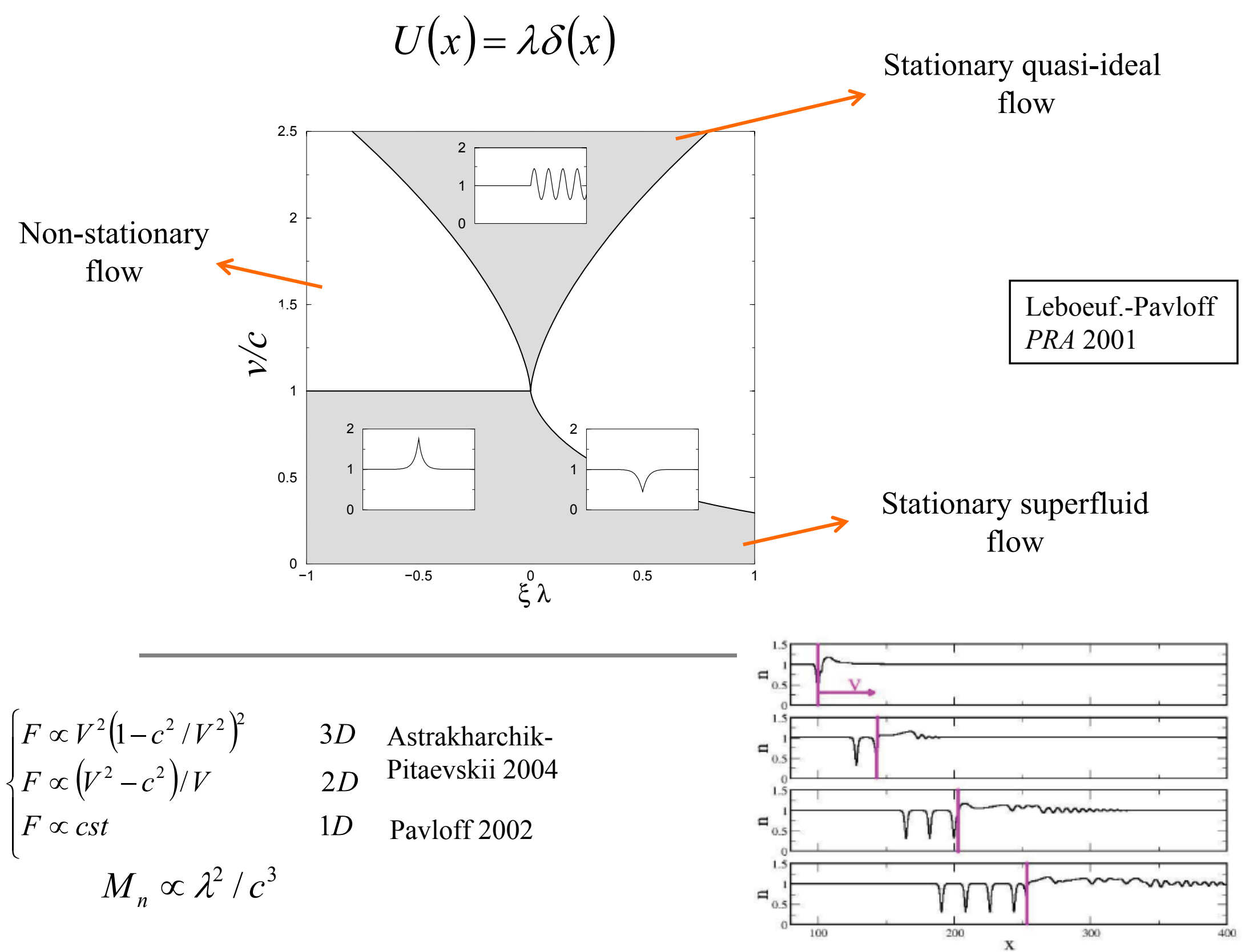




\section{Experimental tests}
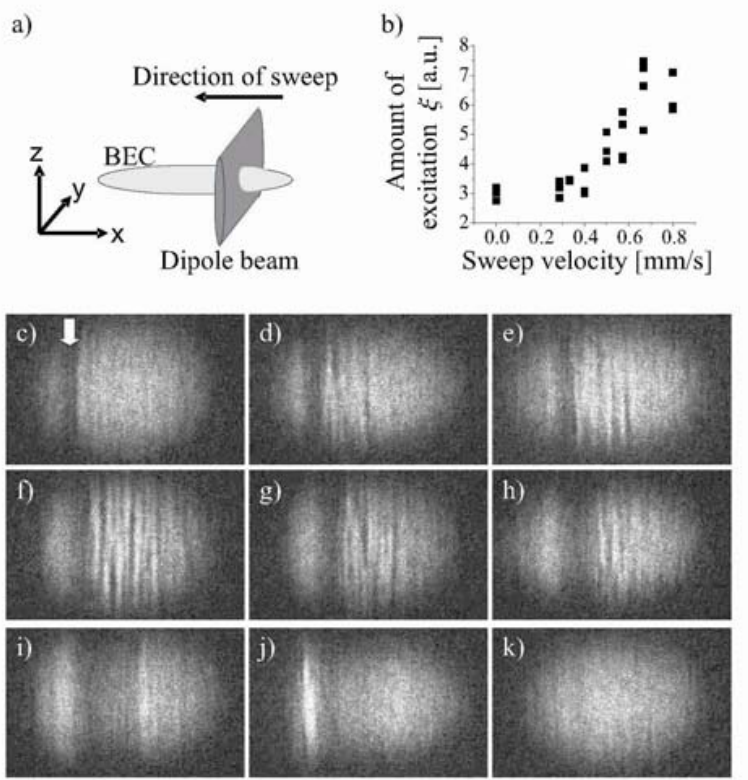

a)

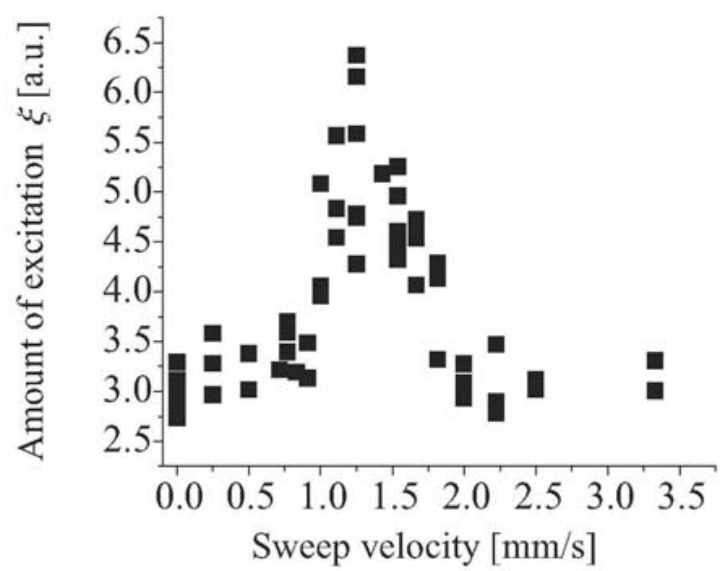

P. Engels, C. Atherton, Phys. Rev. Lett. 99, 160405 (2007)

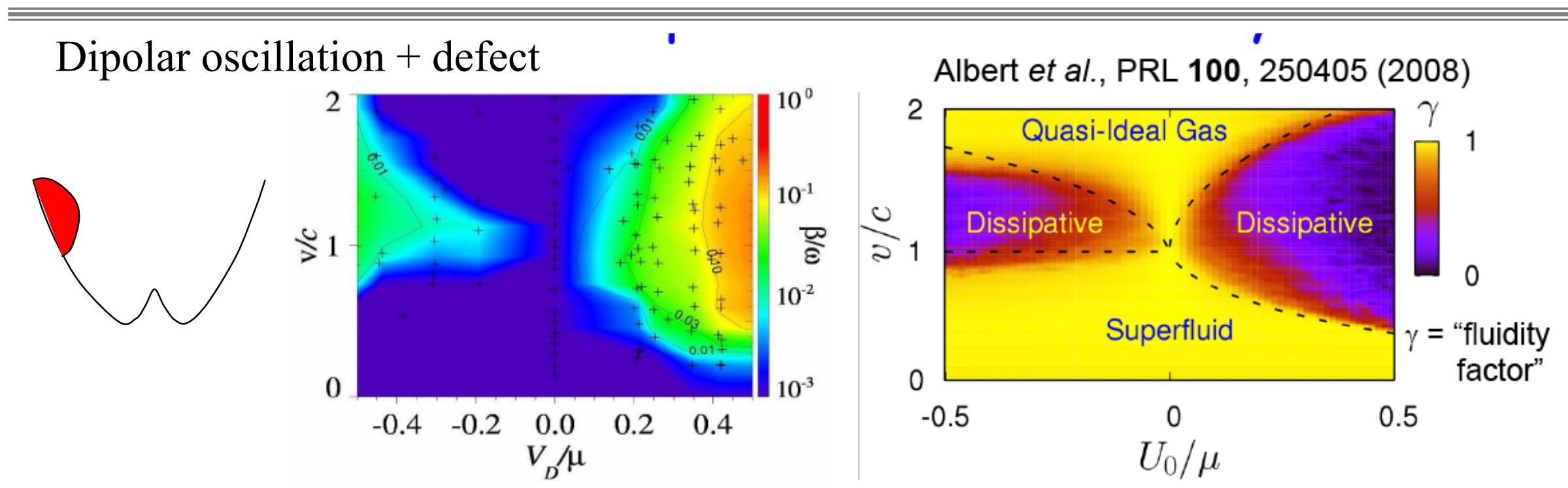

D. Dries, S. E. Pollack, J. M. Hitchcock and R. G. Hulet, arXiv:1004.1891 
Interacting bosons in a random potential: Two contrasting phenomena

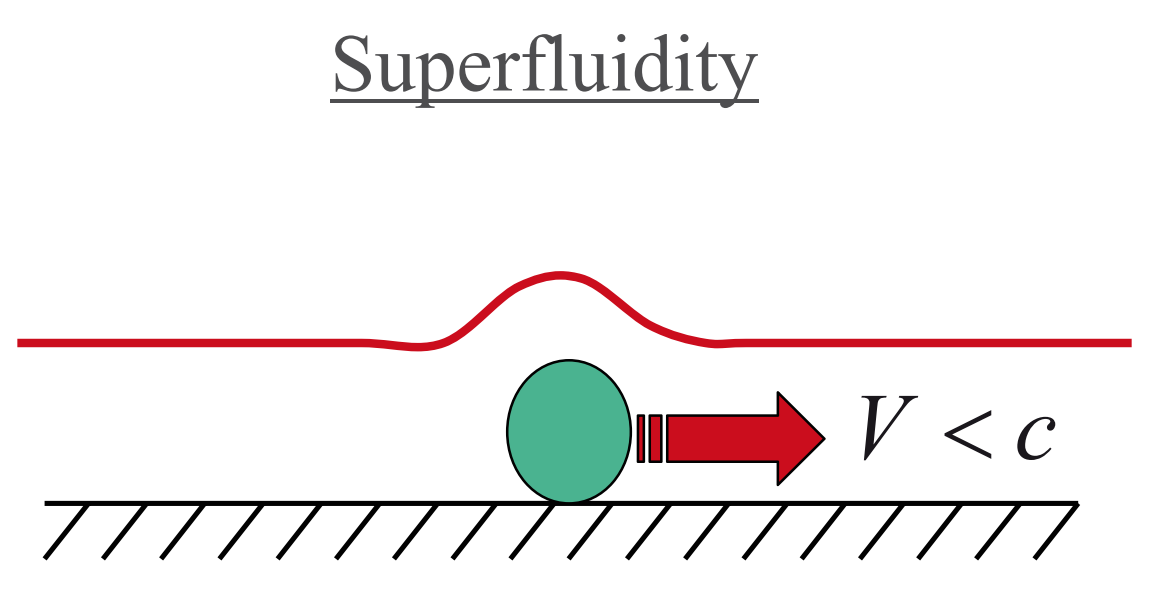

- No dissipation

- No drag force

- Perfect transmission

- Normal fluid fraction Interaction $\prec$ Disorder 


\section{Flow through a random potential}

A series of uncorrelated $\delta$ peaks

$$
U(x)=\frac{\hbar^{2}}{m b} \sum_{n=1}^{N_{i}} \delta\left(x-x_{n}\right)
$$

Correlated Gaussian potential

Speckle potential

Uncorrelated $\delta$ peaks

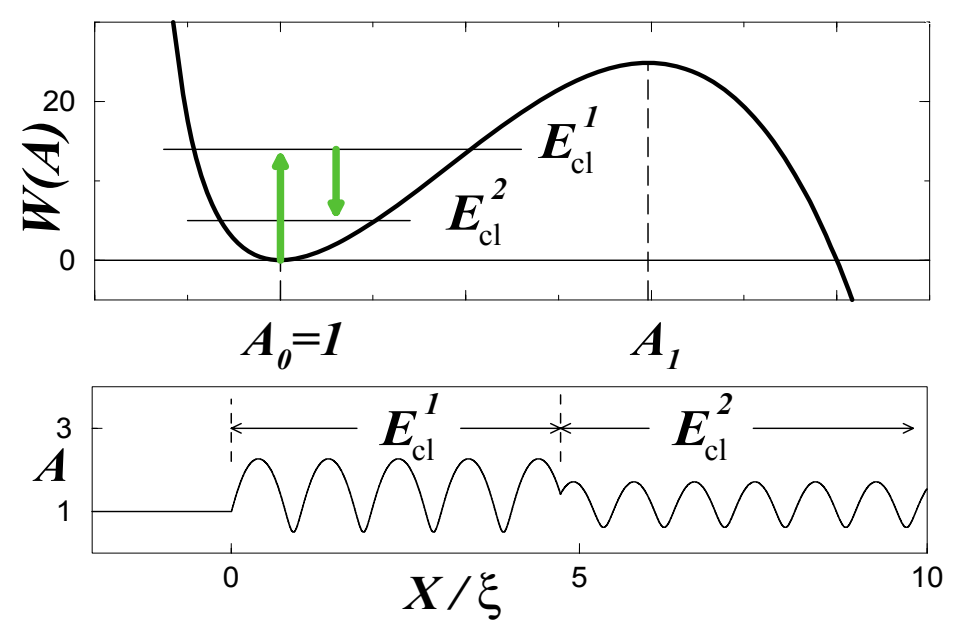

$\circ\langle U\rangle=\hbar^{2} n_{i} /(m b)$

○ $\langle U(x) U(0)\rangle-\langle U\rangle^{2}=\left(\hbar^{2} / m\right)^{2} \sigma \delta(x)$

$$
\left(\langle U\rangle<<\mu+m V^{2} / 2, \quad \sigma=n_{i} / b^{2}, \quad n_{i}=N_{i} / L\right)
$$




\section{$\underline{\text { Summary of Results }}$}

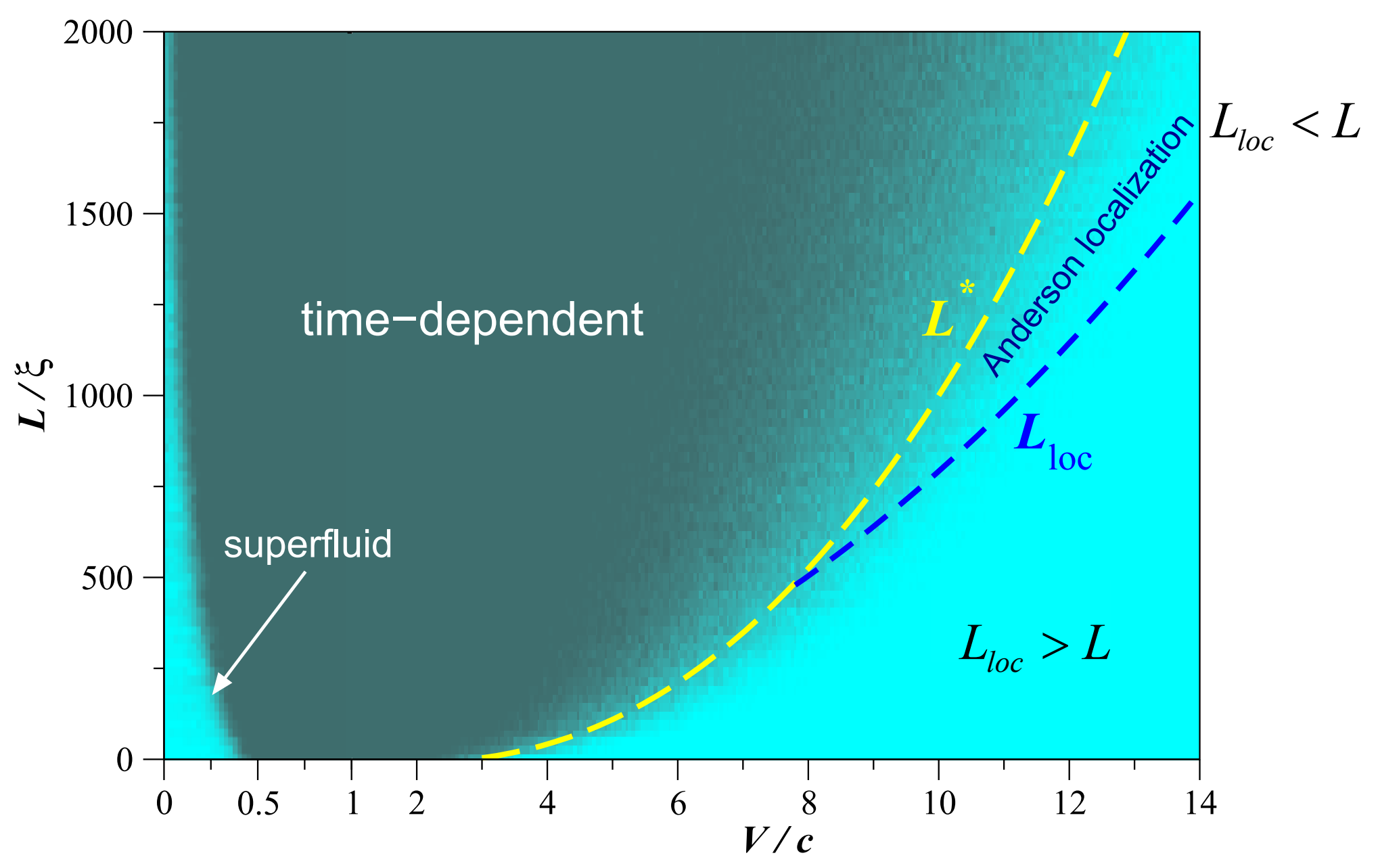

Phys. Rev. Lett. $\underline{98}, 210602$ (2007) 
SF Regime:

Subsonic motion

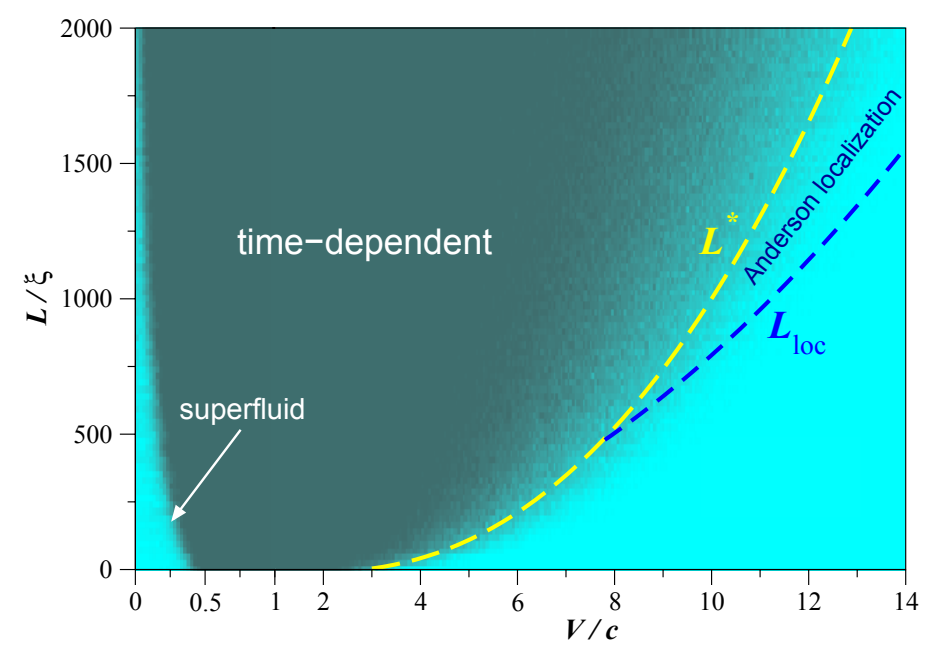

$$
\delta n(X) \cong-\frac{m n_{0}}{\hbar^{2} \kappa} \int_{-\infty}^{\infty} d y U(y) \exp [-2 \kappa|X-y|]
$$

$$
\kappa=\frac{m}{\hbar} \sqrt{\left|c^{2}-V^{2}\right|} \quad \text { Effective wave vector }
$$

$M_{n} / M=\frac{\lambda^{2} n_{i} \xi}{2}(1-V / c)^{-3 / 2}, \quad\left(\kappa>>n_{i}\right)$

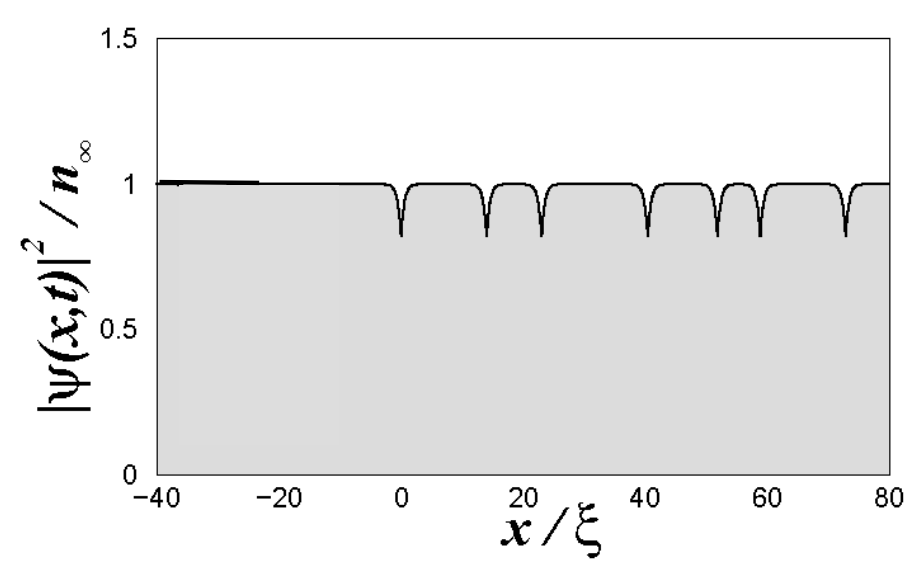

Density locally perturbed in the region of the potential, $\rightarrow$ perfect transmission

$\rightarrow$ no dissipation nor drag exerted on the potential 
Non-perturbative supersonic flow

$$
L_{l o c}(\kappa)<L
$$

2000
$1500-$

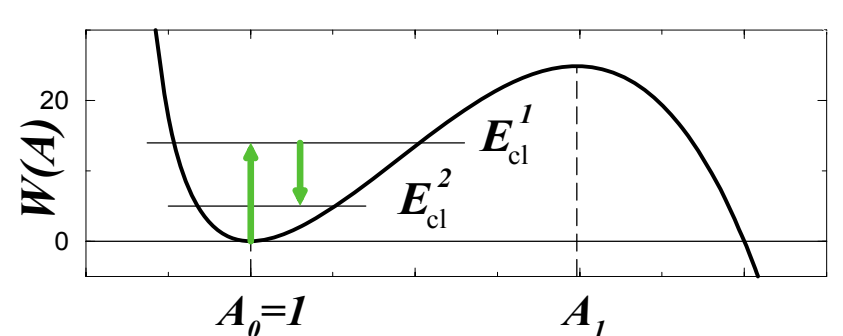

$$
\lambda_{j}=m E_{c l}^{(j)} /\left(2 \hbar^{2} \kappa^{2}\right)^{\stackrel{w}{2}}
$$

$$
P(\lambda, j) d \lambda
$$

time-dependent

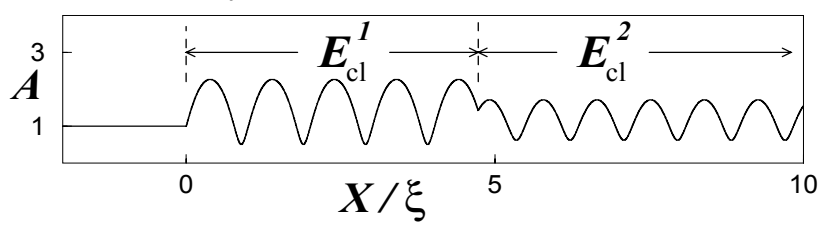

$$
t=j /\left(\kappa^{2} b^{2}\right)
$$

Diffusion process:

$$
\frac{\partial P}{\partial t}=\frac{\partial}{\partial \lambda}\left[\lambda(1+\lambda) \frac{\partial P}{\partial \lambda}\right]
$$

(DMPK Equation) (Dorokov-Mello-Pereyra-Kumar)

$$
\begin{gathered}
\langle\ln T\rangle=-\frac{L}{L_{l o c}(\kappa)} \quad L_{l o c}(\kappa)=\frac{\kappa^{2} / \sigma}{\hat{C}(2 \kappa)} \quad \sqrt{\kappa=\frac{m}{\hbar} \sqrt{\left|V^{2}-c^{2}\right|}} \\
\kappa=\frac{m}{\hbar} \sqrt{\left|V^{2}-c^{2}\right|} \rightarrow \mathrm{k} \quad L_{l o c}(\kappa) \rightarrow L_{l o c}(k) \quad \begin{array}{c}
\text { Antsygina-Pastur-Slyusarev } \\
\text { formula }
\end{array}
\end{gathered}
$$


Uncorrelated delta peaks

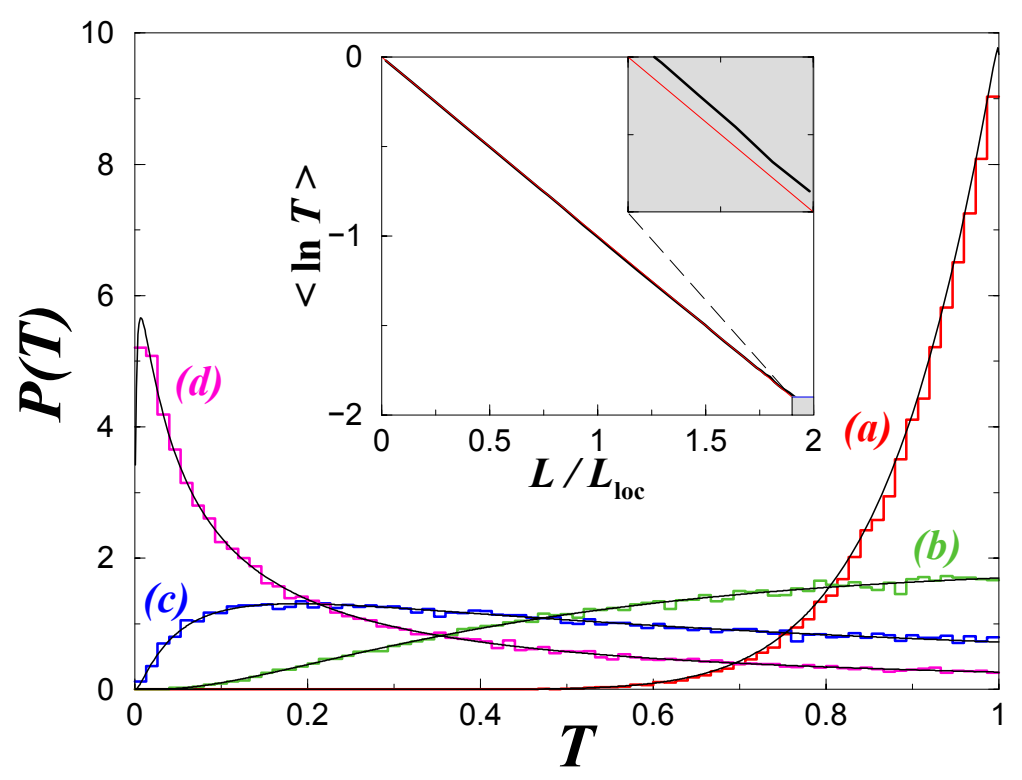

$$
L / L_{l o c}(\kappa)=0.1,0.5,1,2
$$$$
P(T)=\frac{L_{l o c}}{L} \exp \left[-\frac{L_{l o c}}{L}(1-T)\right]
$$$$
P(\ln T)=\sqrt{\frac{L_{l o c}}{4 \pi L}} \exp \left[-\frac{L_{l o c}}{4 L}\left(\frac{L}{L_{l o c}}+\ln T\right)^{2}\right]
$$

Speckle potential

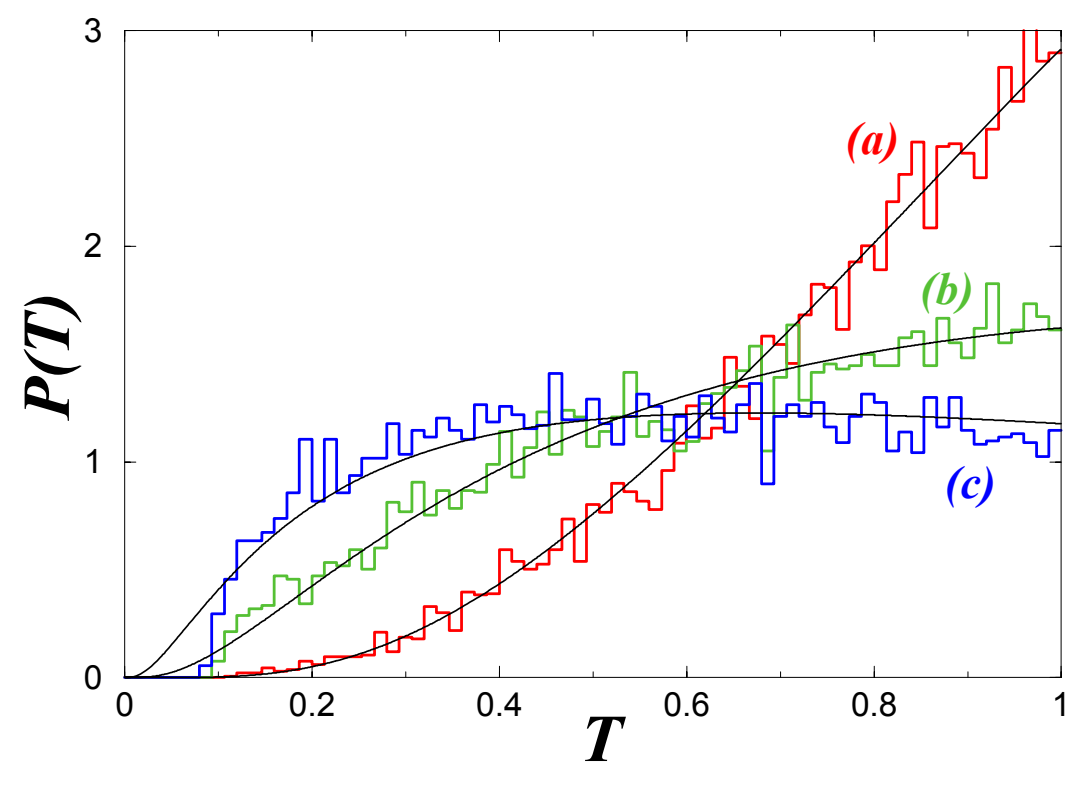

$$
L / L_{l o c}(\kappa)=0.31,0.52,0.68
$$


Crossover to the time-dependent regime
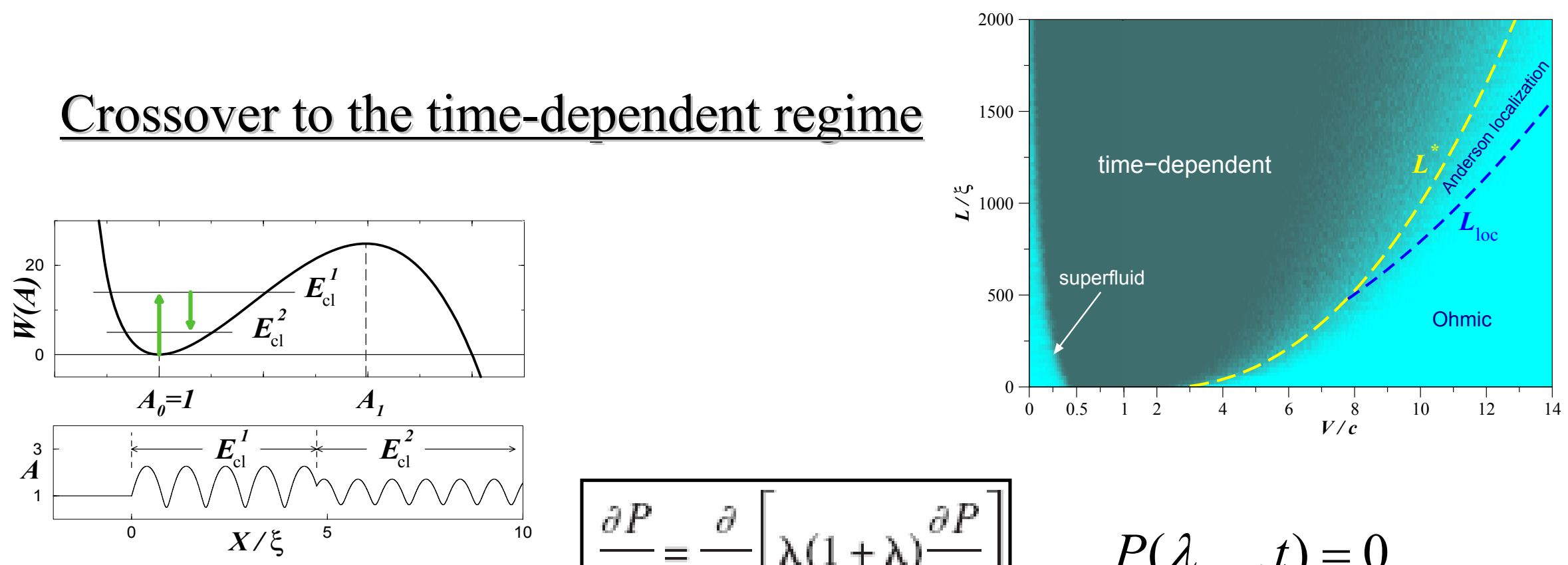

$$
\frac{\partial P}{\partial t}=\frac{\partial}{\partial \lambda}\left[\lambda(1+\lambda) \frac{\partial P}{\partial \lambda}\right] \quad P\left(\lambda_{\max }, t\right)=0
$$
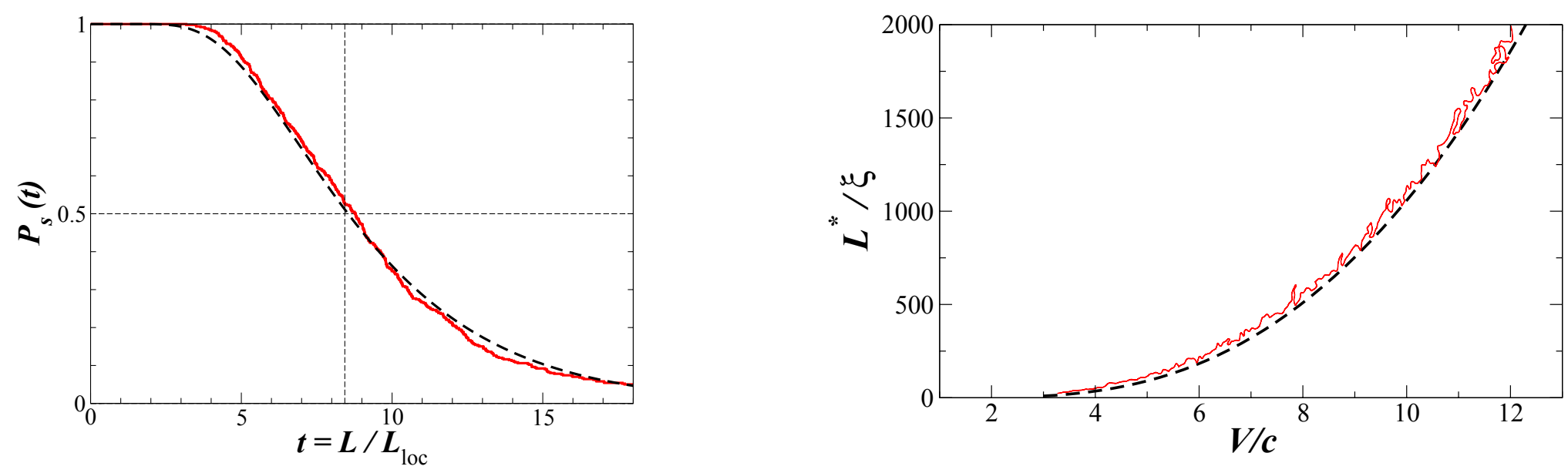

$$
P_{s}\left(t^{*}\right)=1 / 2
$$

$$
L^{*}(\kappa) \approx L_{l o c}(\kappa)\left[\ln \left(\frac{V^{2}}{16 c^{2}}\right)-1\right]
$$




\section{Crossover from superfluid to time-dependent regime: A problem of extreme value statistics}

Slowly varying disordered potential : $\quad \xi / l_{c}<<1$
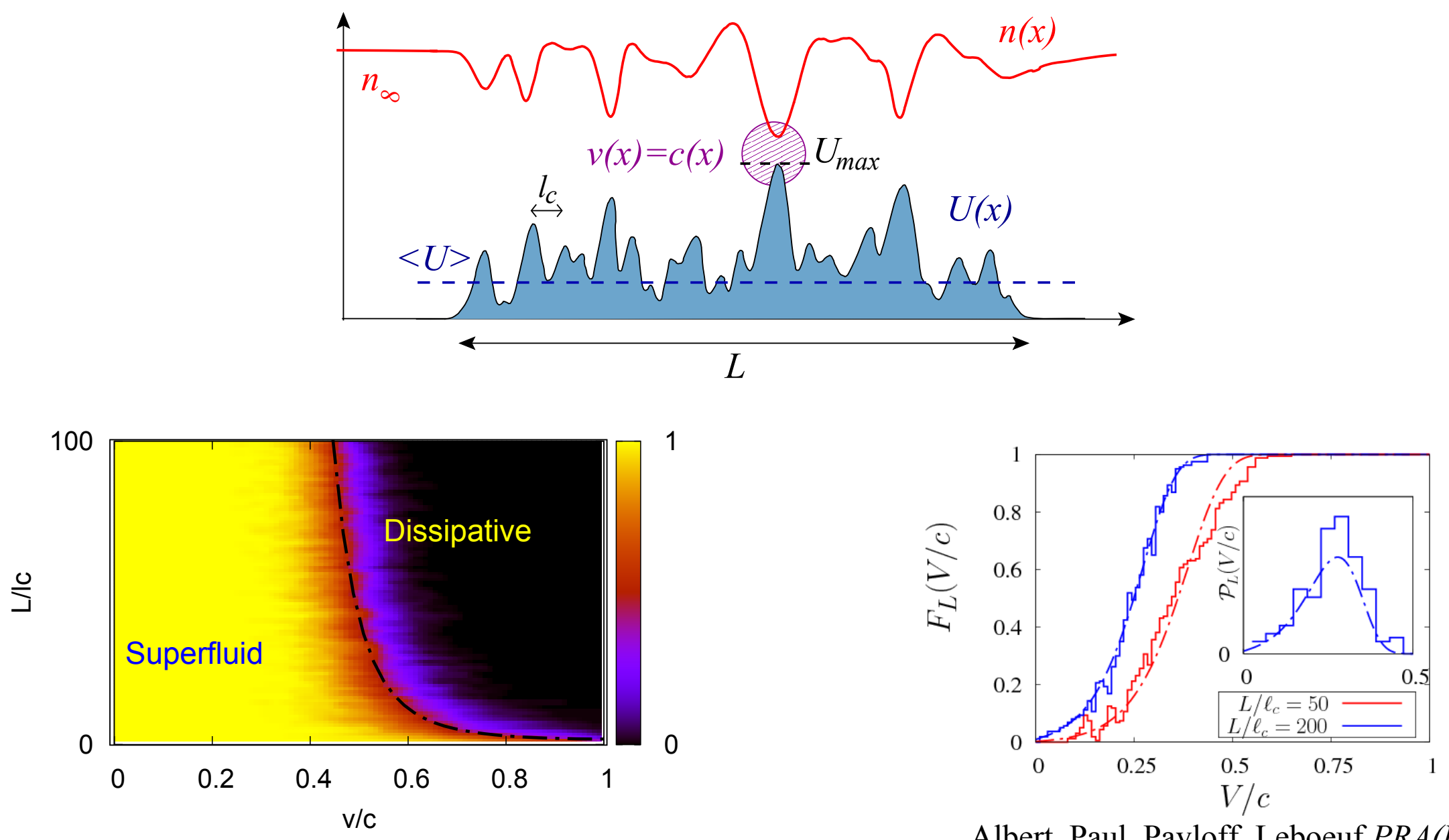

Albert, Paul, Pavloff, Leboeuf PRA(R) 2010 


\section{Experiments with Dipole Oscillations}

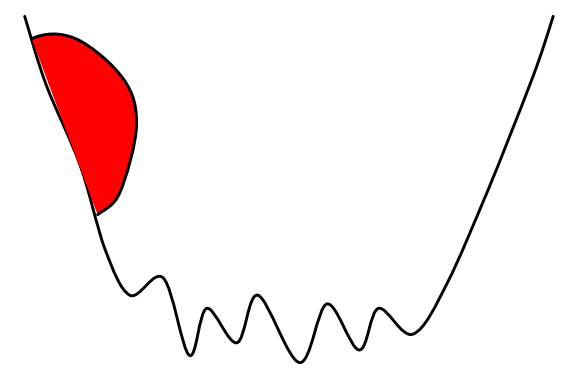

Disordered potential
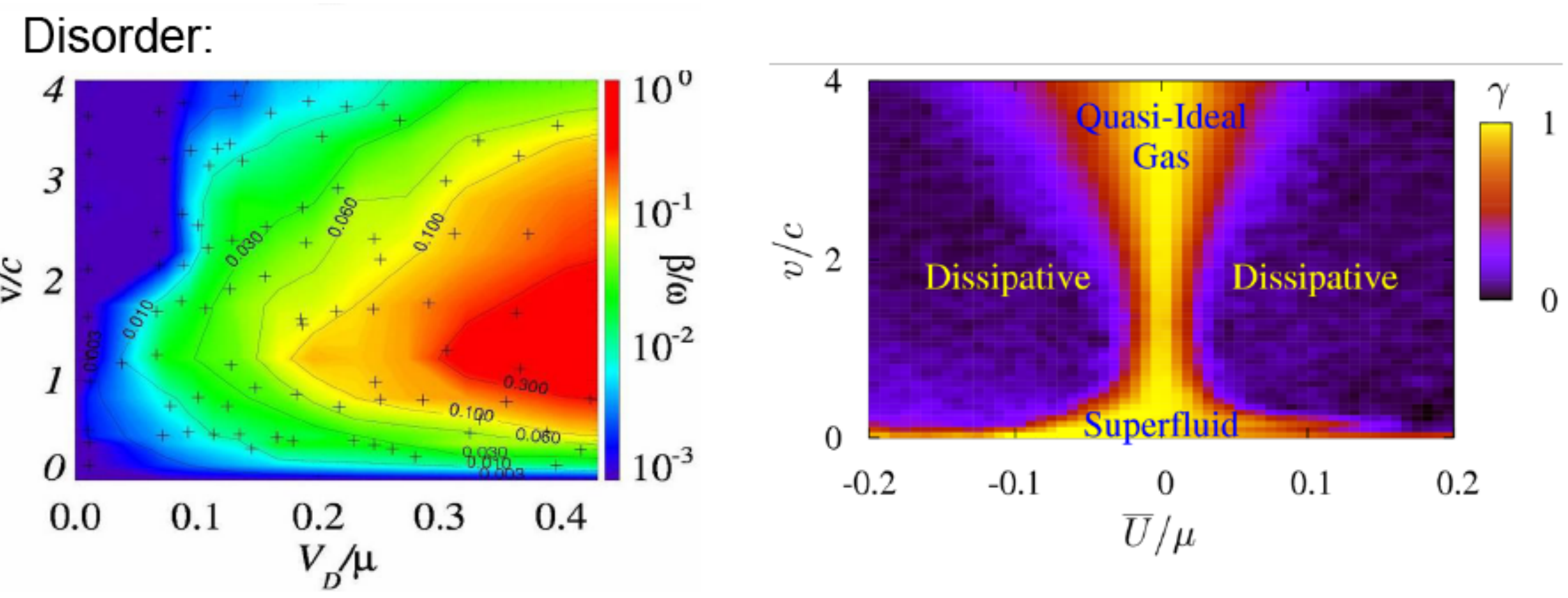

Theory: M. Albert, T. Paul, N. Pavloff, P. Leboeuf, Phys. Rev. Lett. 100, 250405 (2008)

Experiment: D. Dries, S. E. Pollack, J. M. Hitchcock and R. G. Hulet, arXiv:1004.1891. 


\section{Recent experiments on localization with cold atoms}

- Orsay : Billy et al, Nature 2008 : Weakly interacting BEC in a speckle disorder
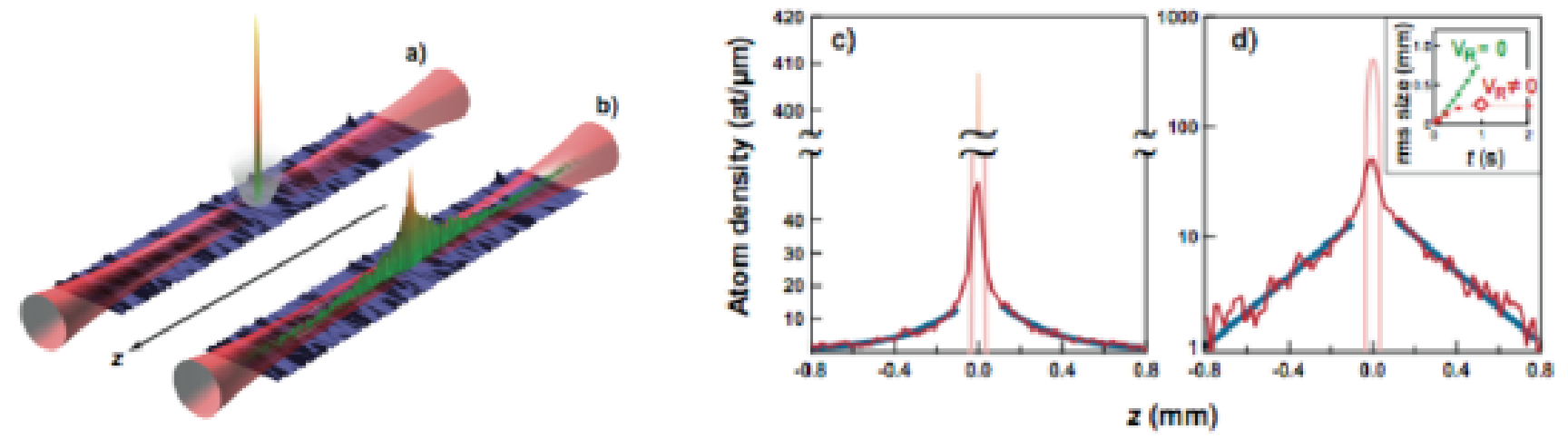

- Firenze : Roati et al, Nature 2008 : Non interacting cold atoms in a quasi-periodic potential

$$
\hat{H}_{\exp }=-\frac{\hbar^{2}}{2 m} \frac{\partial^{2}}{\partial x^{2}}+V_{1} \cos ^{2}\left(k_{1} x\right)+V_{2} \cos ^{2}\left(k_{2} x\right)
$$
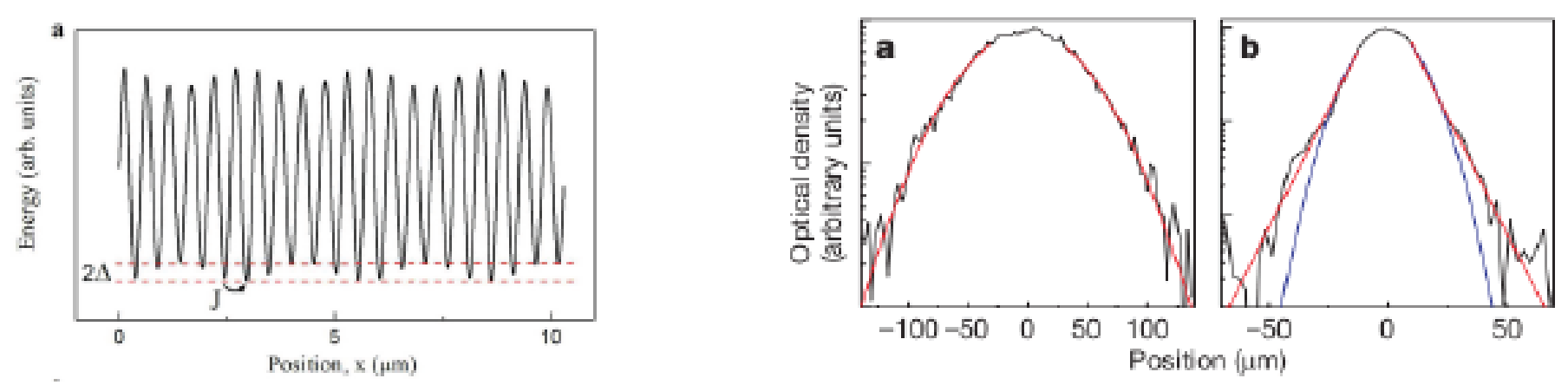

«Half » way between a periodic and a random system 
Anderson localization and nonlinearity in one dimensional disordered photonic lattices

Yoav Lahini ${ }^{1}$, Assaf Avidan ${ }^{1}$, Francesca Pozzi ${ }^{2}$, Marc Sorel ${ }^{2}$, Roberto

Morandotti $^{3}$, Demetrios N. Christodoulides ${ }^{4}$ and Yaron Silberberg ${ }^{1}$

(a)

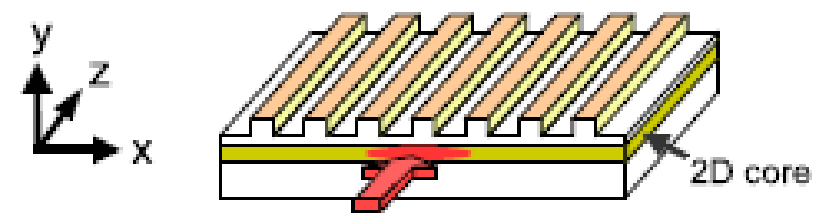

(b)

(c)

(d)

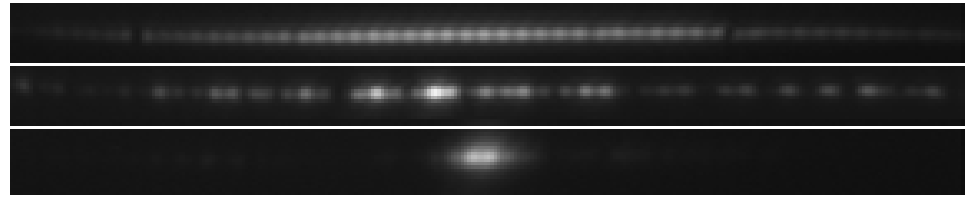

$$
i \frac{\partial A_{k}}{\partial z}=-C\left(A_{k+1}+A_{k-1}\right)+\gamma\left|A_{k}\right|^{2} A_{k}+\epsilon_{k} A_{k}
$$

- $C$ : tunneling rate between adjacent waveguides

- $\varepsilon_{\mathrm{\kappa}}$ : on-site energy

- $\gamma$ : strength of the nonlinearity of the medium $(>0$, self defocusing medium)

- Paraxial approximation

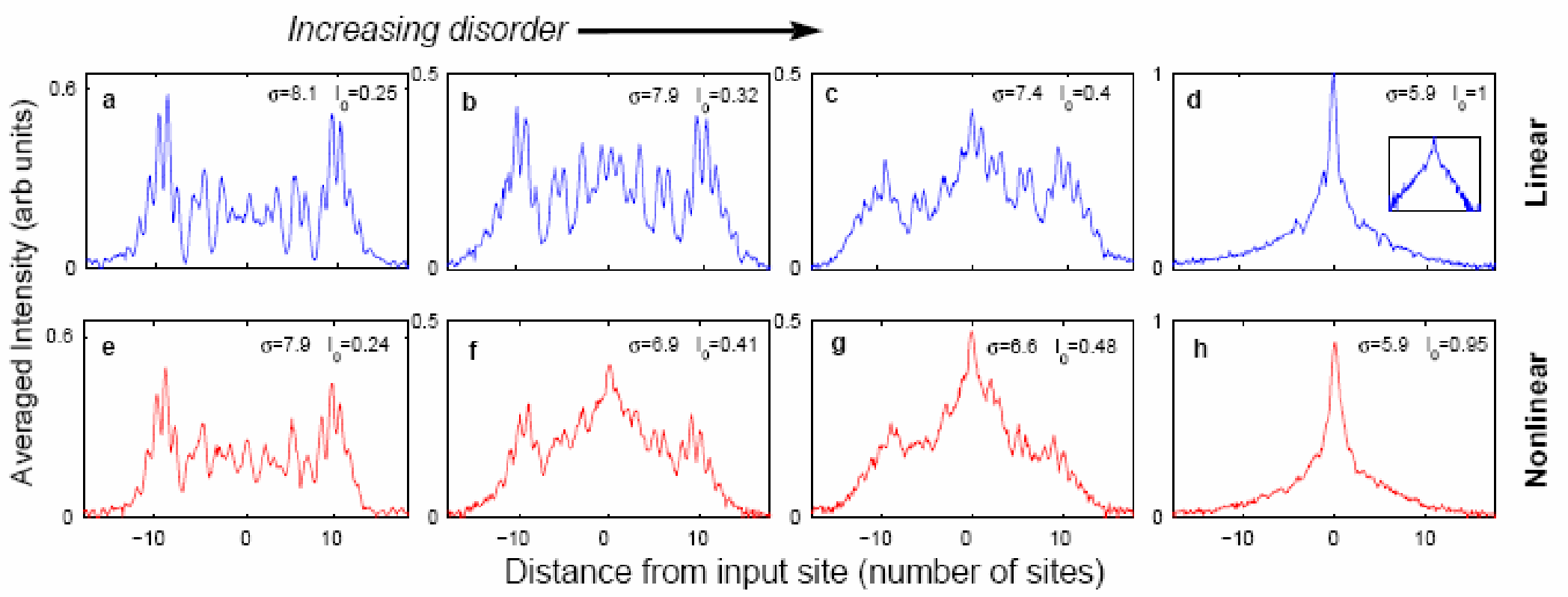




\section{Superfluid Motion of Light}

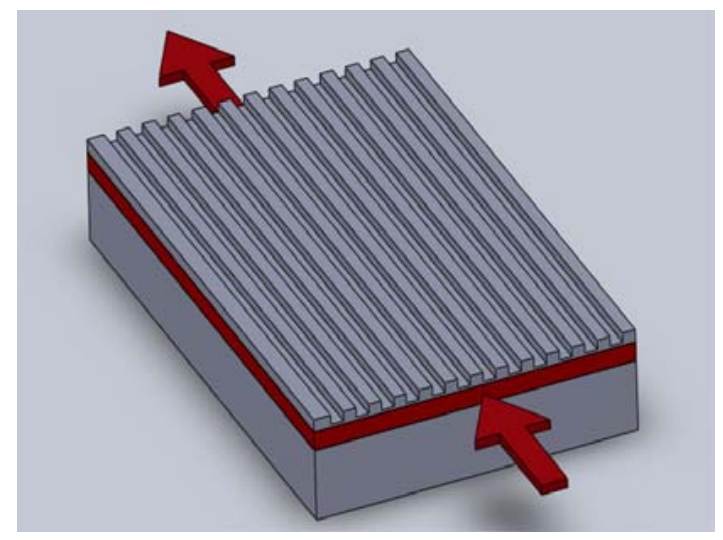

$$
i \frac{\partial A_{k}}{\partial z}=-C\left(A_{k+1}+A_{k-1}\right)+\gamma\left|A_{k}\right|^{2} A_{k}+\epsilon_{k} A_{k}
$$

Non-linear equation, similar to the mean-field description of cold atoms $=>$ does superfluid motion of light exists?

$$
v_{c}^{L} \propto \sqrt{\gamma C|A|^{2}} \approx 2 \times 10^{-2} \quad \text { Critical speed }
$$

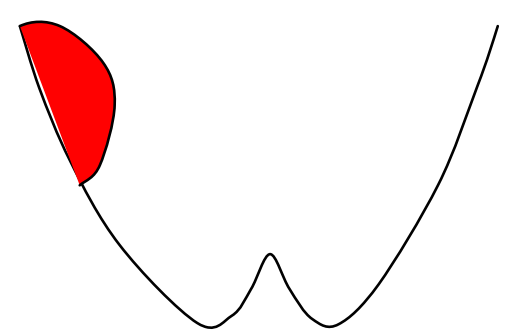

Localized impurity (attractive or repulsive)

$$
\epsilon_{k}=\epsilon_{0}+\frac{1}{2} \omega^{2} k^{2}+U_{0} \delta_{k, 0}
$$


(a)
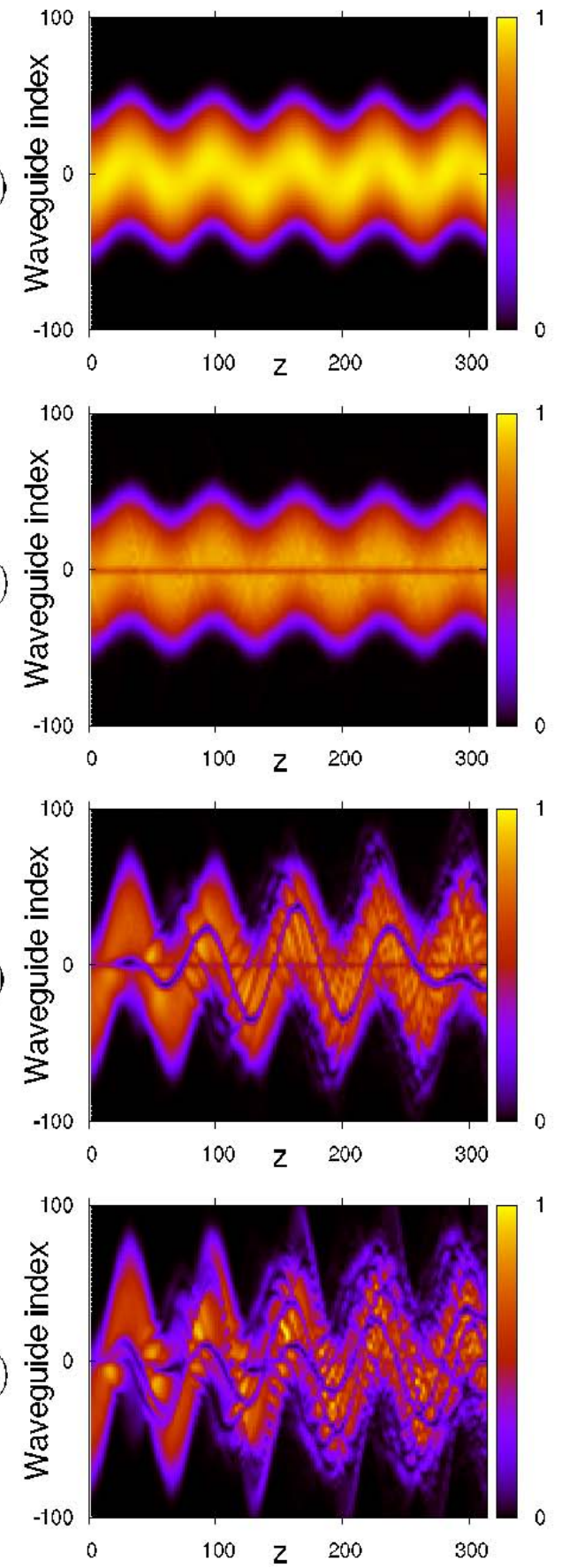
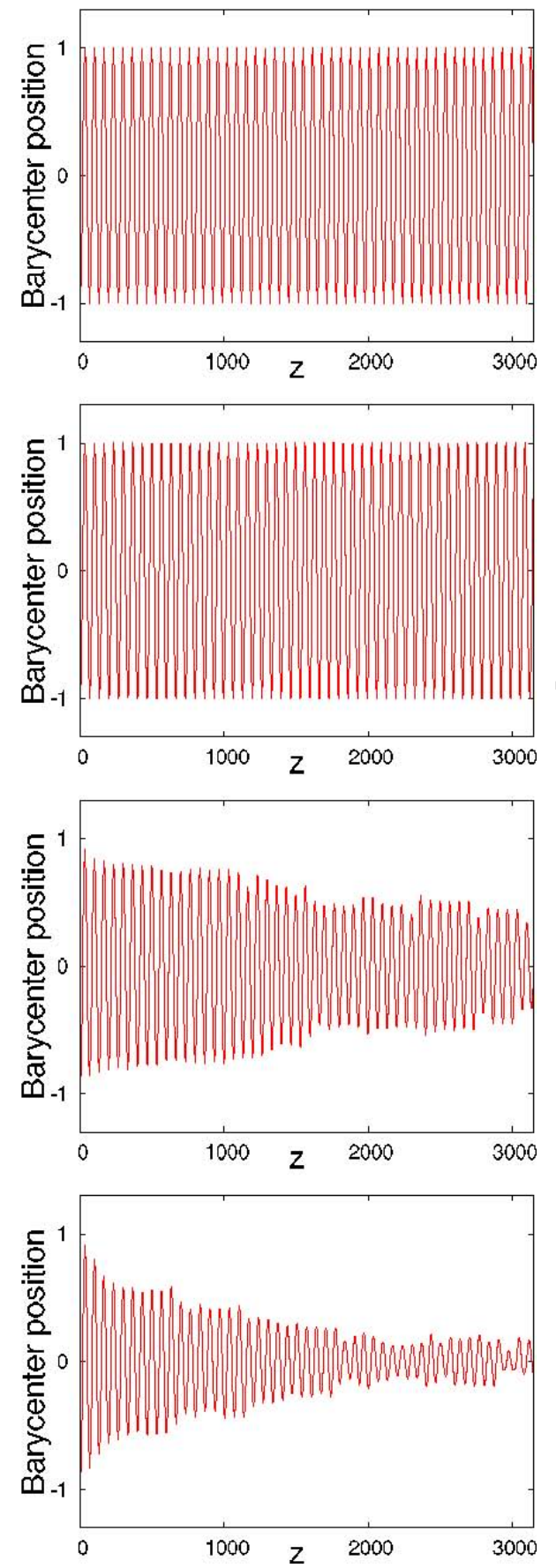

$$
\epsilon_{k}=\epsilon_{0}+\frac{1}{2} \omega^{2} k^{2}+U_{0} \delta_{k, 0}
$$

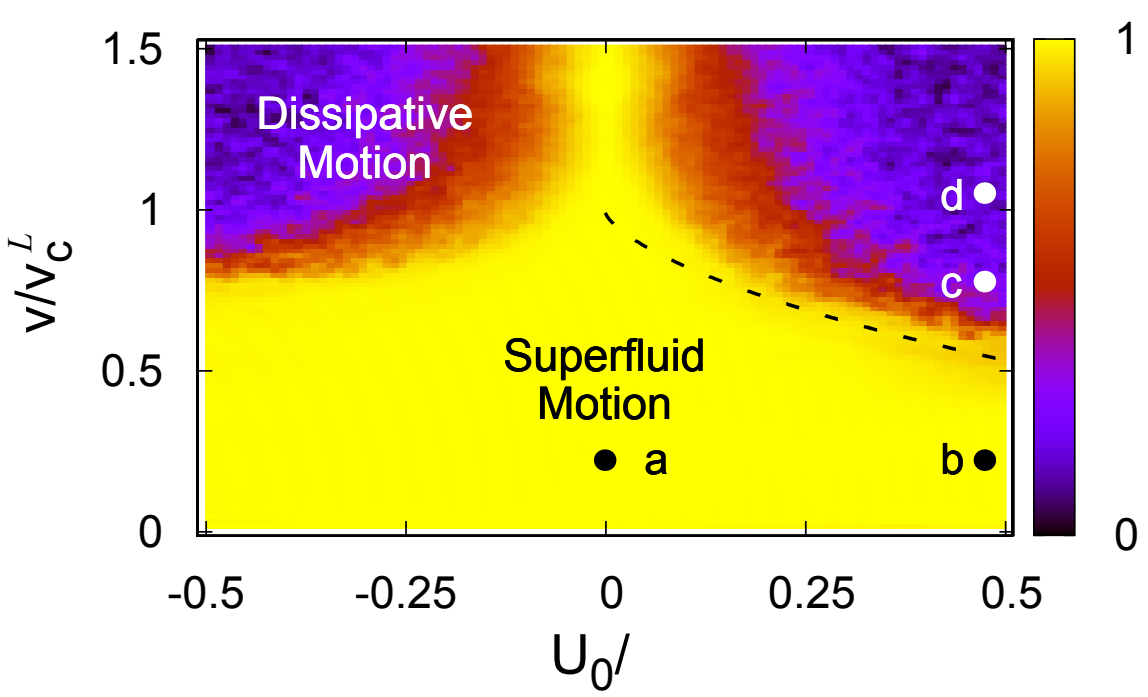

P. Leboeuf, S. Moulieras to appear 


\section{Concluding remarks}

- We provide analytical and numerical evidence of $A L$ in the presence of interaction for \# types of disorder. Generalization of DMPK diffusion equation to include interactions. No localization as L goes to infinity.

- Renormalization of the localization length due to interactions

- The flow is SF at small velocities either for an obstacle or a disordered potential

- Existence of a time-dependent regime, at intermediate velocities or large sample lengths

- Estimates of crossovers to an unstable flow, either from the sub or supersonic regimes

- Dipole oscillations. Experiments on BEC show SF as well as the presence of unstable flow in presence of disorder

- Extension of the concept of superfluidity to motion motion of light in NL media 


\section{Some Related References}

\# Bose-Einstein beams: coherent propagation through a guide

P. Leboeuf, N. Pavloff

Phys. Rev. A 64 (2001) 033602

\# Breakdown of superfluidity of an atom laser past an obstacle

N. Pavloff

Phys. Rev. A 66 (2002) 013610

\# Solitonic transmission of Bose-Einstein matter waves

P. Leboeuf, N. Pavloff, S. Sinha

Phys. Rev. A 68 (2003) 063608

\# Nonlinear transport of Bose-Einstein condensates through waveguides with disorder

T. Paul, P. Leboeuf, N. Pavloff, K. Richter, P. Schlagheck

Phys. Rev. A $\underline{72}, 063621$ (2005)

\# Superfluidity versus Anderson localization in a dilute Bose gas

T. Paul, P. Schlagheck, P. Leboeuf, N. Pavloff

Phys. Rev. Lett. 98, 210602 (2007)

\# Dipole oscillations of a BEC in the presence of defects and disorder

M. Albert, T. Paul, N. Pavloff, P. Leboeuf

Phys. Rev. Lett. 100, 250405 (2008)

\# Anderson localization of a weakly interacting one-dimensional Bose gas

T. Paul, M. Albert, P. Schlagheck, P. Leboeuf, N. Pavloff

Phys. Rev. A $\underline{80}, 033615$ (2009)

\# Localization by bichromatic potentials versus Anderson localization

M. Albert, P. Leboeuf

Phys. Rev. A 81, 013614 (2010)

\# Breakdown of superfluidity of a matter wave in a random environment

M. Albert, T. Paul, N. Pavloff, P. Leboeuf

Phys. Rev. A 82, 011602 (2010)

\# Superfluid motion of light

P. Leboeuf, S. Moulieras

to appear 